\title{
The effectiveness of methotrexate and low-dose steroid therapy in the treatment of idiopathic granulomatous mastitis
}

\author{
Erkan Dalbaşs ${ }^{1, A-F}$, Ömer Lütfi Akgül2,A-F \\ ${ }^{1}$ Memorial Hospital, Department of General Surgery, Diyarbakır, Turkey \\ ${ }^{2}$ Genesis Hospital, Department of General Surgery, Diyarbakır, Turkey \\ A - research concept and design; $B$ - collection and/or assembly of data; $C$ - data analysis and interpretation; \\ $D$ - writing the article; $E$ - critical revision of the article; $F$ - final approval of the article
}

\section{Address for correspondence}

Erkan Dalbaşı

E-mail: erkandalbasi144@gmail.com

Funding sources

None declared

Conflict of interest

None declared

Received on April 15, 2021

Reviewed on July 26,2021

Accepted on August 4, 2021

Published online on September 9, 2021

\begin{abstract}
Background. Idiopathic granulomatous mastitis (IGM) is a rare, chronic, benign, inflammatory breast disease of unknown cause. Patients usually present with a single breast mass, hyperemia, discharge, skin disorders, and fever. Radiological and clinical findings can mimic carcinoma and infection.
\end{abstract}

Objectives. To examine the treatment of IGM with methotrexate (MTX) + low-dose steroid, and present the results and follow-up data from our center.

Materials and methods. Sixty-two patients, diagnosed with IGM in our center between January 2009 and December 2017 were included in this study. Patients diagnosed with granulomatous mastitis histopathologically underwent testing with anamnesis, physical examination and imaging methods to exclude other diseases that cause granulomatous reactions. Patients with a history of malignancy, chronic infectious diseases such as hepatitis B and pregnant women were excluded from this study. Data collected from 62 patients were reviewed retrospectively for this study.

Results. The mean patient age was $36.58 \pm 5.83$ years (range: $28-54$ years). Lesions were present in the right breast in 30 (48.38\%) patients, the left breast in 26 (41.94\%) patients and both breasts in 6 (9.68\%) patients. Methotrexate was administered orally at a dose of $15 \mathrm{mg} /$ week and methylprednisolone at a dose of $8 \mathrm{mg} / \mathrm{day}$. The mean clinical and radiological remission periods of these patients were $10.14 \pm 1.21$ months (range: 3-14 months). All patients attended regular follow-up appointments. The recovery rate of patients during follow-up was determined to be $93.71 \%$.

Conclusions. Methotrexate + low-dose steroid therapy is successful in the treatment of IGM. Prospective, large case series and/or multi-center studies are needed to develop an IGM treatment algorithm.

Key words: autoimmune disease, methotrexate, low-dose steroid, granulomatous mastitis

Cite as

Dalbaşı, E, Akgül Ö. The effectiveness of methotrexate

and low-dose steroid therapy in the treatment of idiopathic granulomatous mastitis. Adv Clin Exp Med.

2021;30(10):1091-1097. doi:10.17219/acem/140842

DOI

10.17219/acem/140842

Copyright

Copyright by Author(s)

This is an article distributed under the terms of the

Creative Commons Attribution 3.0 Unported (CC BY 3.0)

(https://creativecommons.org/licenses/by/3.0/) 


\section{Background}

Idiopathic granulomatous mastitis (IGM) is a rare, chronic, benign, inflammatory breast disease of unknown cause. ${ }^{1}$ It is an idiopathic mastitis that mostly affects young and middle-aged women. Although there is no clear information about its incidence, there are publications stating it to be around $0.37 \%$. Its prevalence is approx. 2.4 per $100,000 .^{2}$ Patients usually present with a single breast mass, hyperemia, discharge, skin disorders, and fever. Radiological and clinical findings can mimic carcinoma and infection. ${ }^{3}$ A definitive diagnosis is made with histopathologic examination. Fine needle aspiration, tru-cut biopsy, or incisional or excisional biopsies can be performed for tissue evaluation. Although chronic inflammation, microabscess and fat necrosis can be seen in tissue biopsies, the presence of non-caseous epithelioid and multinucleated giant cell granulomas within the breast lobules are histopathological findings for IGM. ${ }^{4}$ Even though the pathogenesis of IGM is not known, it is thought to be an autoimmune response to extravasated secretions from the lobules. ${ }^{5}$ The coexistence of other autoimmune diseases such as erythema nodosum and arthritis in IGM patients, and their responsiveness to immunosuppressive drugs support this view. ${ }^{6,7}$ Systemic diseases that cause granulomatous lesions such as mycobacteria, fungal infections, sarcoidosis, Wegener granulomatous, and polyarteritis nodosa should be excluded. ${ }^{8}$ Currently, there are no established treatment algorithms for IGM. Treatment options include antibiotics, immunosuppressive agents, glucocorticoids, and surgical procedures used alone or in combination. ${ }^{9}$

\section{Objectives}

Methotrexate (MTX) reversibly inhibits dihydrofolate reductase, preventing the conversion of folic acid to tetrahydrofolic acid. The inhibition of tetrahydrofolate results in the cessation of adenine-guanine synthesis and suppresses cell proliferation through the inhibition of protein synthesis. ${ }^{10}$ Methotrexate treatment remission rates are around 60-90\% and recurrence rates are lower than those of surgical and steroid therapy. ${ }^{11}$ This study aims to examine the treatment results of MTX + low-dose steroid and followup data in patients diagnosed with IGM in General Surgery Department of Memorial Hospital (Diyarbakır, Turkey).

\section{Materials and methods}

\section{Patient follow-up}

Sixty-two female patients diagnosed with IGM in our center between January 2009 and December 2017 were included in the study. The mean age of participants was $36.58 \pm 5.83$ years (range: $28-54$ years). Patients diagnosed with granulomatous mastitis histopathologically underwent testing with anamnesis, physical examination and imaging methods to exclude other diseases that cause granulomatous reactions. Patients with a history of malignancy, chronic infectious diseases such as hepatitis B and pregnant women were excluded from the study. Data collected from 62 patients were reviewed retrospectively for this study. The study protocol was approved by the ethics committees at Memorial Hospital (Diyarbakır, Turkey) in agreement with the Declaration of Helsinki of 1975. All participants provided voluntary informed consent prior to the initiation of the study. The demographic data of the patients, presence of comorbidities, symptoms on hospital admission, laboratory tests, radiological imaging, microbiological culture results, MTX and methylprednisolone doses and durations, surgical history, and the presence of recurrence were recorded. The possibility of malignancy was excluded using radiological and pathological examination. Tru-cut biopsy was performed for pathological examination and abscesses present in patients were aspirated, and the contents were

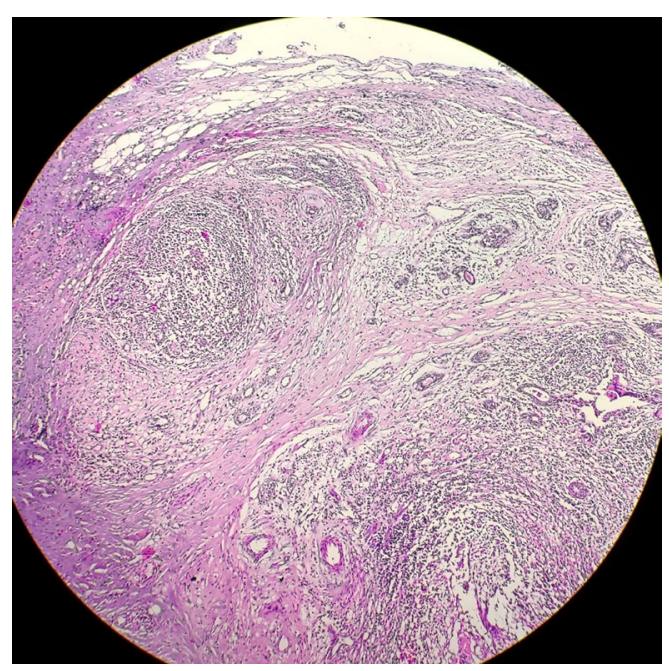

Fig. 1. Hematoxylin and eosin (H\&E) staining of idiopathic granulomatous mastitis (IGM)

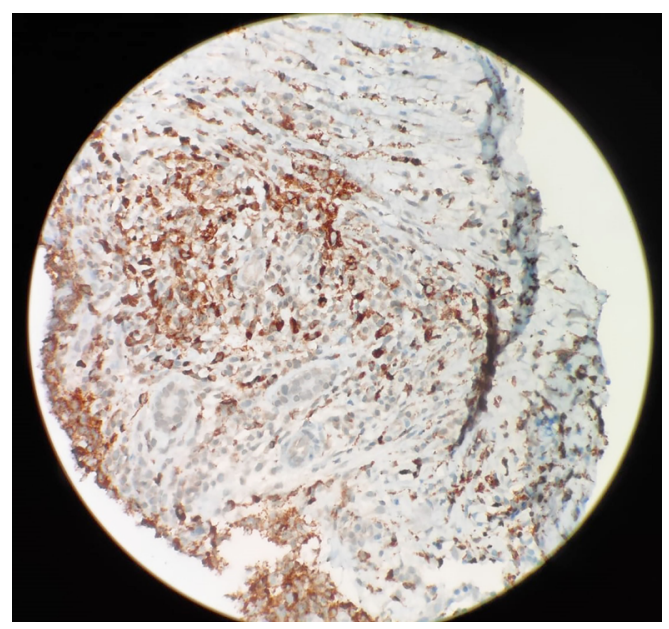

Fig. 2. CD68 immunohistochemical staining of idiopathic granulomatous mastitis (IGM) 


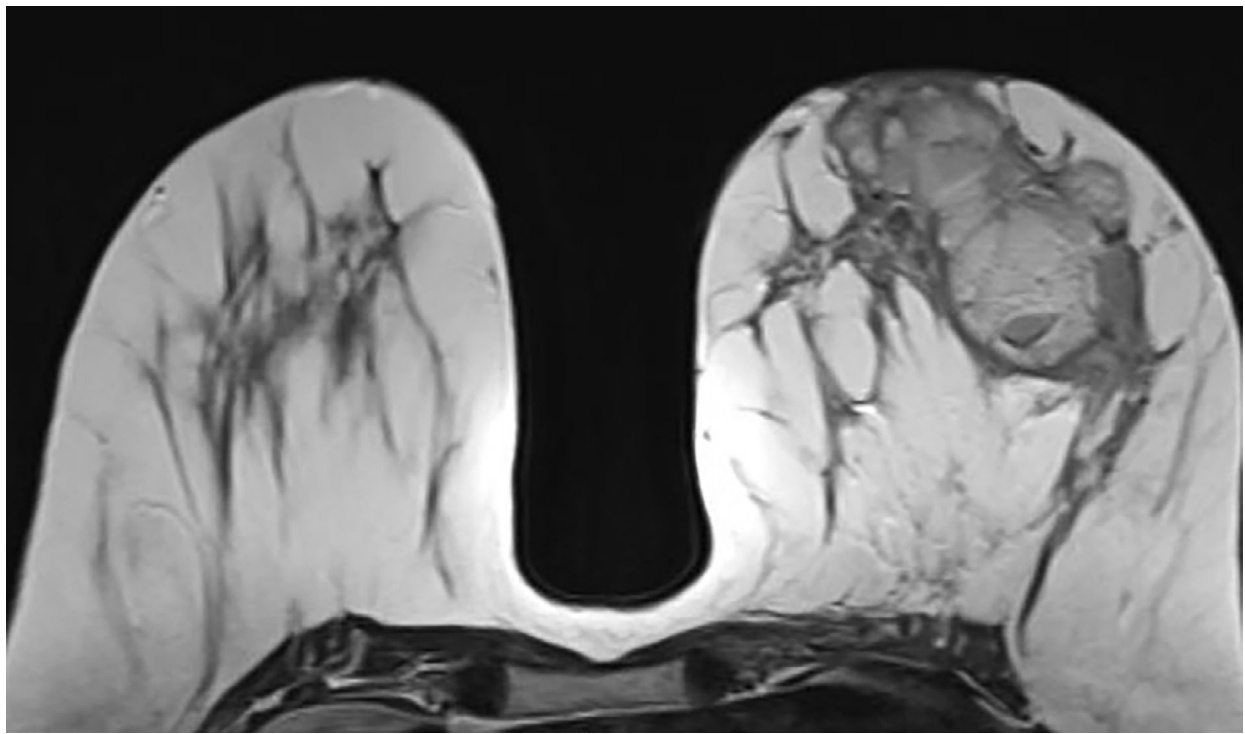

Fig. 3. Magnetic resonance image (MRI) of idiopathic granulomatous mastitis (IGM)

sent for microbiological culture. The CD68 was used for immunostaining. A microscopic image of IGM with hematoxylin and eosin (H\&E) staining is shown in Fig. 1 and CD68 immunostaining in Fig. 2. Cultures were performed to rule out bacterial infections, tuberculosis and fungal infections. Symptoms before and after the treatment as well as laboratory results were compared. Radiological imaging with bilateral breast axillary ultrasonography (USG) and breast magnetic resonance imaging (MRI) were performed in all patients. An MRI image of IGM is shown in Fig. 3. Patients' responses to treatment were evaluated using USG.

After the diagnosis of IGM, oral treatment with $8 \mathrm{mg} /$ day of methylprednisolone and $15 \mathrm{mg} /$ week of MTX started.

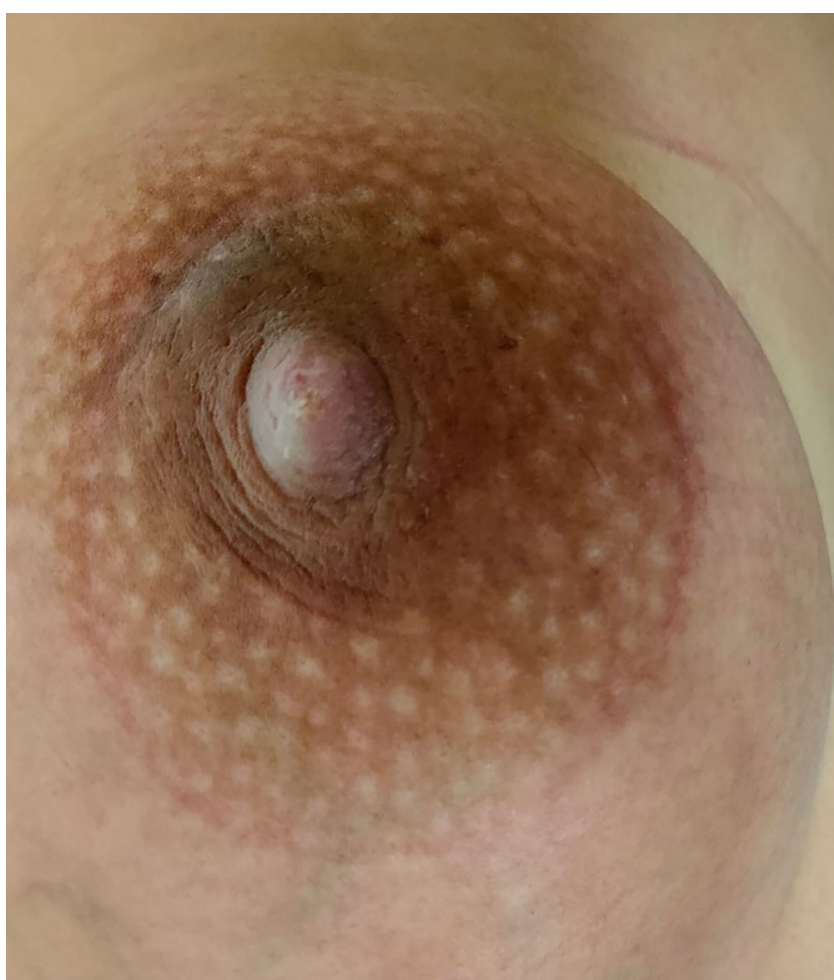

Fig. 4. First application image of idiopathic granulomatous mastitis (IGM)
Subcutaneous MTX treatment was started in patients who could not tolerate or did not respond to oral MTX and the dose was increased to $25 \mathrm{mg} /$ week. Calcium, vitamin $\mathrm{D}$, folic acid, and a proton pump inhibitor were administered to protect against the side effects of steroids and MTX. The patients were followed up every 3 weeks with systemic examination, complete blood count, and liver and kidney function tests. The treatment period of patients after remission was planned as 24 months.

Erythrocyte sedimentation rate (ESR), C-reactive protein (CRP) and white blood cell (WBC) count were measured before starting treatment. In addition, clinical findings such as mass size and pain severity were compared before and after the remission.

In our study, remission was defined as the regression of inflammatory changes in the breast, closure of fistulas, healing of existing skin lesions, and regression of USG findings. Recurrence of symptoms during or after treatment was deemed to be a relapse. Photographs of a patient's breasts at the time of diagnosis (Fig. 4) and after the treatment (Fig. 5) are shown.

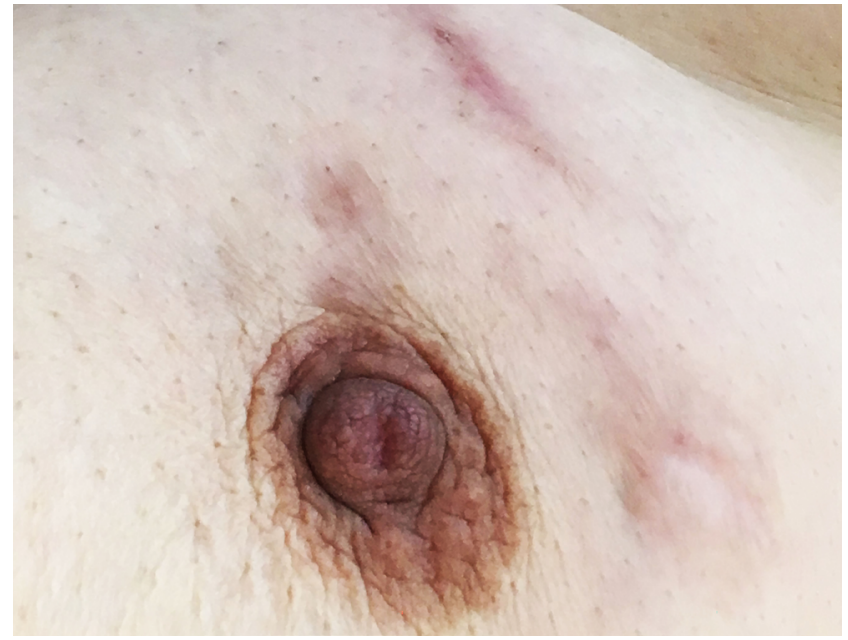

Fig. 5. Healed skin fistula of idiopathic granulomatous mastitis (IGM) 


\section{Immunohistochemical staining}

Heat-induced antigen retrieval was performed using a microwave oven (700 W'; Bosch, Gerlingen, Germany) for $3 \mathrm{~min}$ at $90^{\circ} \mathrm{C}$. The heating process was performed with a citrate buffer ( $\mathrm{pH} 6)$ solution for proteolysis. Sections were washed in phosphate-buffered saline (PBS) $(3 \times 5 \mathrm{~min})$ and incubated with hydrogen peroxide (K-40677109; Merck, Darmstadt, Germany) for $15 \mathrm{~min}$. Other sections were washed in PBS $(3 \times 5 \mathrm{~min})$ and blocked with Ultra V Block (lot \# PHL150128; Thermo Fisher Scientific, Waltham, USA) for $8 \mathrm{~min}$. After draining, CD68 Monoclonal Antibodies (KP1), (cat \# MA5-13324, 1:100-1:200; Thermo Fisher Scientific) were applied directly to the sections, which were then incubated, left overnight at $4^{\circ} \mathrm{C}$, then washed in PBS $(3 \times 5 \mathrm{~min})$ and incubated with Biotinylated Secondary Antibody (lot \# PHL150128; Thermo Fisher Scientific) for 20 min. After washing with PBS, Streptavidin Peroxidase (lot \# PHL150128; Thermo Fisher Scientific) was applied to the sections for $15 \mathrm{~min}$. The sections were again washed in PBS $(3 \times 5 \mathrm{~min})$ and DAB (lot \# HD36221, Thermo Fisher Scientific) was applied to the sections for up to $10 \mathrm{~min}$. The sections were counterstained in Harris's Hematoxylin for $45 \mathrm{~s}$. Then, they were dehydrated in a graded series of alcohols and cleared in xylene. Slides were mounted with Entellan and examined under a light microscope (Carl Zeiss AG, Jena, Germany).

\section{Statistical analyses}

Statistical analyses were performed using the IBM SPSS v. 22.0 (IBM Corp., Armonk, USA) software. Results are presented as mean \pm standard deviation. Statistical differences before and after treatment were evaluated using a paired t-test. A $X^{2}$ test was used to ensure homogeneity of variances in the group. A value of $\mathrm{p}<0.05$ was considered significant.

\section{Results}

During follow-up of patients, chest radiographs and liver and kidney function tests were normal. Growth occurred in microbiological cultures in 23 (37.09\%) patients. Staphylococcus aureus was identified in 9 patients, Streptococcus in 7 patients, and Corynebacterium in 7 patients. Despite the appropriate antibiotic therapy for 10 days, based on culture antibiogram results, the patient's symptoms did not regress.

\section{Histopathological findings}

The diagnosis of IGM was confirmed with histopathology. In histopathological examination, epithelioid histiocytes, giant cells, fibroblasts, and reactive ductal cells were detected with standard H\&E staining. Nucleolus prominence, cell nucleus growth and hyperchromasia seen in epithelioid cells can suggest malignancy. In addition, the cohesive nature of the cells can be confused with other proliferative lesions. Therefore, the diagnosis was supported by the presence of immunopositive staining of epithelioid cells, Langerhans-type giant cells, and histiocytic cells with CD68 markers.

\section{Clinical findings}

Lesions were present in the right breast in 30 (48.38\%) patients, in the left breast in $26(41.94 \%)$ patients and in both breasts in $6(9.68 \%)$ patients. Lesions were located in the upper quadrants in 38 (61.29\%) patients while 21 (33.87\%) patients had lesions in the lower quadrants. Widespread involvement was present in 3 (4.83\%) patients. The difference in terms of right and left breast involvement was statistically significant $(\mathrm{p}<0.001)$. All patients experienced pain, breast mass and hyperemia in the affected area. Erythema nodosum and arthralgia were associated with IGM in 7 (11.29\%) and $4(6.45 \%)$ patients, respectively. No patients had a diagnosis of concomitant chronic disease. Axillary lymphadenopathy was seen in 26 (41.93\%) patients. Post-treatment lymphadenopathy was seen in $4(6.45 \%)$ patients. Fistulas and purulent discharges were observed in 15 (24.19\%) patients, and ulceration of the breast skin in 9 (14.51\%). After treatment, all fistulas and ulcers healed. Five (8.06\%) patients developed nipple collapse prior to treatment. After the treatment, the nipple remained collapsed despite disease recovery in 1 (1.61\%) of these patients. Three (4.83\%) patients were nulliparous. None of the patients were pregnant or lactating at the time of diagnosis. Twenty (32.25\%) patients were using oral contraceptives. The body mass index (BMI) of $38(61.29 \%)$ patients was over $30 \mathrm{~kg} / \mathrm{cm}^{2}$. Thirty-one (50\%) patients were active smokers. The demographic information and clinical findings are summarized in Table 1.

Methotrexate was administered orally at a dose of $15 \mathrm{mg} /$ week and methylprednisolone at $8 \mathrm{mg} /$ day. In 5 (8.06\%)

Table 1. Demographic and clinical characteristics

\begin{tabular}{|l|c|}
\hline \multicolumn{1}{|c|}{ Variable } & Value \\
\hline Age [years] & $36.58 \pm 5.83$ \\
\hline $\begin{array}{l}\text { Mass location, } \mathrm{n}(\%) \\
\text { right breast } \\
\text { left breast } \\
\text { bilateral }\end{array}$ & $30(48.38)$ \\
Clinical findings, $\mathrm{n}(\%)$ & $26(41.94)$ \\
erytema nodosum & $6(9.68)$ \\
arthralgia & \\
fistula & $7(11.29)$ \\
ulceration & $4(6.45)$ \\
nipple collapse & $15(24.19)$ \\
axillary lymphadenopathy & $9(14.51)$ \\
\hline Smoker, $\mathrm{n}$ (\%) & $5(8.06)$ \\
\hline Follow-up duration [months] & $26(41.93)$ \\
\hline Oral contraceptives, $\mathrm{n}$ (\%) & $31(50)$ \\
\hline
\end{tabular}


patients who could not tolerate oral MTX, the same dose was administered subcutaneously and steroids were continued. Clinical improvement was observed in 47 (75.80\%) patients within the first 3 months. The mean clinical and radiological remission periods of the patients were $10.14 \pm 1.21$ months (range: $3-14$ months). Due to the lack of clinical improvement in 15 (24.19\%) patients within the first 3 months, the MTX dose was gradually increased to $25 \mathrm{mg} /$ week and subcutaneous administration was initiated. Additionally, the dose of methylprednisolone was increased to $12 \mathrm{mg} /$ day. In 10 of those 15 patients, remission was achieved after the increase in the dosage of the treatment medications. Surgery was performed in 5 patients who failed to achieve remission. A segmental mastectomy was performed to excise the entire cavity. After surgery, MTX and methylprednisolone treatment was begun at the initial dose, and remission was achieved in all patients. The mean ESR of the patients before treatment was $48.25 \pm 23.84 \mathrm{~mm} / \mathrm{h}$ and decreased to $14.34 \pm 10.82 \mathrm{~mm} / \mathrm{h}$ after treatment. The mean CRP value was $32.34 \pm 24.23 \mathrm{mg} / \mathrm{L}$ before treatment. After treatment, the CRP value dropped to $4.68 \pm 3.5 \mathrm{mg} / \mathrm{L}$. The WBC was $18.64 \pm 5.301000 / \mu \mathrm{L}$ before treatment and $6.41 \pm 2.141000 / \mu \mathrm{L}$ after treatment. The mean size of the radiological mass measured $42.32 \pm 4.21 \mathrm{~mm}$ at the time of the $1^{\text {st }}$ application and $8.65 \pm 4.56 \mathrm{~mm}$ after treatment. Comparisons of these differences were made using the values at 6 months post-treatment. All findings were statistically significant $(\mathrm{p}<0.001)$. Changes in the clinical and laboratory data of patients at the $1^{\text {st }}$ admission and after treatment are summarized in Table 2. None of the patients discontinued the medications due to side effects. After achieving remission, all patients continued their treatment and regular follow-ups. The mean duration of treatment was $14 \pm 1.25$ months (range: $6-24$ months). No relapses were observed in any of the patients during the treatment period. Recurrence was observed in a total of 7 (11.29\%) patients -2 patients within the first 3 months, 3 patients within 6 months and 2 patients within a year after the cessation of treatment. One of the recurrences occurred in a patient who underwent surgery. All patients attended regular followed up appointments. The mean follow-up time was $24 \pm 3.6$ months (range: $18-36$ months). The recovery rate of patients during follow-up was determined to be $93.71 \%$.

\section{Discussion}

Idiopathic granulomatous mastitis is a rare, benign breast disease of unknown etiology. ${ }^{12}$ Despite IGM being a disease that seriously affects young women, there has been no standard treatment protocol or remission criteria developed. Factors that lead to increased intraductal secretions such as lactation, prolactinoma, medications, obesity, and smoking are implicated in the etiology. ${ }^{13,14}$ Drainage or surgical resection alone has not been sufficient to cure the disease without the medical treatment. ${ }^{15,16}$ Idiopathic granulomatous mastitis can show similar clinical findings to breast cancer, such as breast skin deterioration and nipple collapse. In addition, culture and histopathologic testing should be performed to rule out other systemic diseases that can cause granulomatous lesions. Therefore, a biopsy was performed for tissue culture and histopathological evaluation in all patients of our study. ${ }^{17}$

Radiological imaging alone is insufficient to confirm the diagnosis of IGM. However, USG and MRI can provide important information on IGM. Numerous combined, irregular, hypoechoic areas on USG indicate the formation of abscesses. In addition, USG can evaluate the axillary lymph nodes for involvement. Parenchymal disorder and heterogeneous lesions with hyperintense signals on MRI images suggest IGM. ${ }^{18}$ In this study, bilateral breast-axillary USG and breast MRI were performed in all patients at the time of diagnosis, and USG was performed to access lesions during follow-up appointments. Although the diagnosis of IGM is confirmed using histopathology, there is no consensus on its treatment. Surgical treatment, antibiotics, steroids, MTX, and immunosuppressive drugs such as azathioprine are options in the treatment of IGM. ${ }^{19}$ Successful results have been achieved with the use of long-term and high-dose steroid therapy. However, the significant side effects such as diabetes, obesity and Cushing's disease experienced with high-dose steroid treatment make this option less desirable. However, the recurrence rate is unacceptably high in low-dose steroid therapy. Studies have reported successful results with MTX monotherapy or concomitant low-dose steroid therapy in attempts to avoid these side effects. ${ }^{20}$ Surgical treatment without medication has a high recurrence and it has been abandoned in the treatment of IGM. ${ }^{21}$

Table 2. Pre- and post-treatment laboratory and clinical findings

\begin{tabular}{|l|c|c|c|}
\multicolumn{1}{|c|}{ Parameters } & Pre-treatment & Post-treatment & p-value \\
\hline ESR $[\mathrm{mm} / \mathrm{h}]$ & $48.25 \pm 23.84$ & $14.34 \pm 10.82$ & $<0.001^{*}$ \\
\hline CRP $[\mathrm{mg} / \mathrm{L}]$ & $32.34 \pm 24.23$ & $4.68 \pm 3.5$ & $<0.001^{*}$ \\
\hline WBC $[1000 / \mu \mathrm{L}]$ & $18.64 \pm 5.30$ & $6.41 \pm 2.14$ & $<0.001^{*}$ \\
\hline Mass size $[\mathrm{mm}]$ & $42.32 \pm 4.21$ & $8.65 \pm 4.56$ & 2.142 \\
\hline Lymphadenopathy, $\mathrm{n}(\%)$ & $15(24.19)$ & $4(6.45)$ & $<0.001^{*}$ \\
\hline Nipple collapse, $\mathrm{n}(\%)$ & $5(8.06)$ & $1(1.61)$ & 2.121 \\
\hline
\end{tabular}

ESR - erythrocyte sedimentation rate; CRP - C-reactive protein; WBC - white blood cell count; * paired t-test. 
The MTX dosage has been started at 10-15 $\mathrm{mg} /$ week and increased to $20-25 \mathrm{mg}$, according to clinical response. This treatment regimen was continued for an average duration of 13-15 months (1-30 months) and resulted in $94 \%$ recovery and $75 \%$ remission in patients at the end of 15 months. These recovery and remission rates are in line with the rates seen in our study. ${ }^{22}$

Kehribar et al. ${ }^{23}$ treated 33 IGM patients with low-dose steroids and 7.5-15 mg/week of MTX. The complete remission rate achieved was $87.8 \%$, and no relapse occurred during the follow-up. In this study, steroid treatment was used for 6-8 weeks, and then monotherapy with MTX was continued. The remission rates found are similar to our findings, but MTX and low-dose steroids were used together for the entire treatment period in our study. It has been reported in studies that MTX reduces IGM recurrence, prevents side effects by decreasing the dose of steroids, and prolongs the remission period without medication. These findings are consistent with those found in our study. $1,24,25$

There are studies showing that lactation is the main factor that causes IGM, and that recurrent pregnancy and breastfeeding lead to relapse. ${ }^{1,2,14}$ In accordance with our findings, $95.17 \%$ of the patients conceived and breastfed at least once. There are studies indicating that oral contraceptive drugs are a risk factor for IGM by increasing intraductal secretion. Oltean et al. ${ }^{26}$ showed that chronic mastitis and oral contraceptive drug use are related. The rate of oral contraceptive use in our study was $32.25 \%$, which is consistent with the literature. There are studies showing smoking and obesity to be controllable causes of IGM. 13,26 Uysal et al. stated that smoking was also effective on IGM on relapse. Recurrence was more common in smokers with IGM. ${ }^{2}$ There is not enough evidence in the literature to prove that obesity causes IGM. ${ }^{2,27}$ On the other hand, in our study, the BMI of 38 (61.29\%) patients was over 30.

A study by Tekgöz et al. ${ }^{13}$ was instrumental in determining the treatment protocol with MTX. It involved $41 \mathrm{pa}-$ tients with IGM. Clinical improvement was observed in all patients within the $1^{\text {st }}$ month of treatment. A remission rate of almost $80 \%$ was achieved. Methotrexate was switched to azathioprine in 4 of the 5 patients who developed recurrence, and in 1 patient who could not tolerate MTX, and all of these patients achieved remission. The patients included in this study did not use any medications other than MTX and steroids. The remission rates achieved in this study are also consistent with our findings.

There are not enough data in the literature to draw definitive conclusions about the role of bacteria in the etiology of IGM. The antibiotic treatment administered based on the culture antibiogram results did not affect remission rates. In many instances, microorganisms identified in cultures are highly likely the results of contamination. ${ }^{28}$

In a study by Ringsted et al., ${ }^{29}$ the results of various treatment methods were evaluated in 28 patients with IGM. Remission without recurrence was achieved in $80 \%$ of patients treated with MTX, $66 \%$ of patients treated with surgery and steroids, and $42 \%$ of patients treated with steroids alone. In this study, arthralgia was seen in $14 \%$ of patients and erythema nodosum in $18 \%$. According to the current literature, the remission rate of IGM patients treated with MTX is around $90 \%$ on average. While the remission rates are similar to our findings, the incidence of additional findings such as arthralgia were higher.

\section{Limitations}

The main limitation of our study is its retrospective nature. Multi-center, prospective studies are needed to demonstrate the efficacy of MTX + low-dose steroid in the treatment of IGM.

\section{Conclusions}

Idiopathic granulomatous mastitis is a rare benign breast disease with unknown etiology or treatment algorithm. Immunosuppressive drugs have been used in its treatment. Although the data are limited, effective results have been obtained with MTX in the treatment of IGM, whether used alone or in combination with steroids. As seen in our study, MTX + low-dose steroid therapy are successful in the treatment of IGM. However, prospective large case series and multi-center studies are needed to definitively determine an IGM treatment algorithm.

\section{ORCID iDs}

Erkan Dalbaşı (D) https://orcid.org/0000-0002-4652-1747

Ömer Lütfi Akgül (D) https://orcid.org/0000-0002-7858-454X

\section{References}

1. Sheybani F, Sarvghad M, Naderi H, Gharib M. Treatment for and clinical characteristics of granulomatous mastitis. Obstet Gynecol. 2015; 125(4):801-807. doi:10.1097/AOG.0000000000000734

2. Uysal E, Soran A, Sezgin E; Granulomatous Mastitis Study Group. Factors related to recurrence of idiopathic granulomatous mastitis: What do we learn from a multicenter study? ANZ J Surg. 2018;88(6): 635-639. doi:10.1111/ans.14115

3. Seo HR, Na KY, Yim HE, et al. Differential diagnosis in idiopathic granulomatous mastitis and tuberculous mastitis. J Breast Cancer. 2012; 15(1):111-118. doi:10.4048/jbc.2012.15.1.111

4. Helal TE, Shash LS, Saad El-Din SA, Saber SM. Idiopathic granulomatous mastitis: Cytologic and histologic study of 65 Egyptian patients. Acta Cytol. 2016;60(5):438-444. doi:10.1159/000448800

5. Patel RA, Strickland P, Sankara IR, Pinkston G, Many W Jr, Rodriguez M. Idiopathic granulomatous mastitis: Case reports and review of literature. J Gen Intern Med. 2010;25(3):270-273. doi:10.1007/s11606-0091207-2

6. Wong SCY, Poon RWS, Chen JHK, et al. Corynebacterium kroppenstedtii is an emerging cause of mastitis especially in patients with psychiatric illness on antipsychotic medication. Open Forum Infect Dis. 2017;4(2):ofx096. doi:10.1093/ofid/ofx096

7. Alibi S, Ferjani A, Gaillot O, Marzouk M, Courcol R, Boukadida J. Identification of clinically relevant Corynebacterium strains by Api Coryne, MALDI-TOF-mass spectrometry and molecular approaches. Pathol Biol (Paris). 2015;63(4-5):153-157. doi:10.1016/j.patbio.2015.07.007

8. Shah KK, Pritt BS, Alexander MP. Histopathologic review of granulomatous inflammation. J Clin Tuberc Other Mycobact Dis. 2017;7:1-12. doi:10.1016/j.jctube.2017.02.001 
9. Barreto DS, Sedgwick EL, Nagi CS, Benveniste AP. Granulomatous mastitis: Etiology, imaging, pathology, treatment, and clinical findings. Breast Cancer Res Treat. 2018;171(3):527-534. doi:10.1007/s10549-0184870-3

10. Strober BE, Menon K. Folate supplementation during methotrexate therapy for patients with psoriasis. J Am Acad Dermatol. 2005;53(4): 652-659. doi:10.1016/j.jaad.2005.06.036

11. Azlina AF, Ariza Z, Arni T, Hisham AN. Chronic granulomatous mastitis: Diagnostic and therapeutic considerations. World J Surg. 2003, 27(5):515-518. doi:10.1007/s00268-003-6806-1

12. Oran EŞ, Gürdal SÖ, Yankol Y, et al. Management of idiopathic granulomatous mastitis diagnosed by core biopsy: A retrospective multicenter study. Breast J. 2013;19(4):411-418. doi:10.1111/tbj.12123

13. Tekgöz E, Çolak S, Çinar M, Yilmaz S. Treatment of idiopathic granulomatous mastitis and factors related with disease recurrence. Turk J Med Sci. 2020;50(5):1380-1386. doi:10.3906/sag-2003-93

14. Taylor GB, Paviour SD, Musaad S, Jones WO, Holland DJ. A clinicopathological review of 34 cases of inflammatory breast disease showing an association between corynebacteria infection and granulomatous mastitis. Pathology. 2003;35(2):109-119. PMID:12745457

15. Pandey TS, Mackinnon JC, Bressler L, Millar A, Marcus EE, Ganschow PS. Idiopathic granulomatous mastitis: A prospective study of 49 women and treatment outcomes with steroid therapy. Breast J. 2014;20(3): 258-266. doi:10.1111/tbj.12263

16. Aghajanzadeh M, Hassanzadeh R, Alizadeh Sefat S, et al. Granulomatous mastitis: Presentations, diagnosis, treatment and outcome in 206 patient from the north of Iran. Breast. 2015;24(4):456-460. doi:10.1016/j.breast.2015.04.003

17. Ergin AB, Cristofanilli M, Daw H, Tahan G, Gong Y. Recurrent granulomatous mastitis mimicking inflammatory breast cancer. BMJ Case Rep. 2011;2011:bcr0720103156. doi:10.1136/bcr.07.2010.3156

18. Gautier N, Lalonde L, Tran-Thanh D, et al. Chronic granulomatous mastitis: Imaging, pathology and management. Eur J Radiol. 2013; 82(4):e165-e175. doi:10.1016/j.ejrad.2012.11.010
19. Akbulut S, Arikanoglu Z, Senol A, et al. Is methotrexate an acceptable treatment in the management of idiopathic granulomatous mastitis? Arch Gynecol Obstet. 2011;284(5):1189-1195. doi:10.1007/s00404-0101825-2

20. Hovanessian Larsen LJ, Peyvandi B, Klipfel N, Grant E, lyengar G. Granulomatous lobular mastitis: Imaging, diagnosis, and treatment. AJR Am J Roentgenol. 2009;193(2):574-581. doi:10.2214/AJR.08.1528

21. Skandarajah A, Marley L. Idiopathic granulomatous mastitis: A medical or surgical disease of the breast? ANZ J Surg. 2015;85(12):979-982. doi:10.1111/ans.12929

22. Postolova A, Troxell ML, Wapnir IL, Genovese MC. Methotrexate in the treatment of idiopathic granulomatous mastitis. J Rheumatol. 2020;47(6):924-927. doi:10.3899/jrheum.181205

23. Kehribar DY, Duran TI, Polat AK, Ozgen M. Effectiveness of methotrexate in idiopathic granulomatous mastitis treatment. Am JMed Sci. 2020;360(5):560-565. doi:10.1016/j.amjms.2020.05.029

24. Kim J, Tymms KE, Buckingham JM. Methotrexate in the management of granulomatous mastitis. ANZ J Surg. 2003;73(4):247-249. doi:10. 1046/j.1445-1433.2002.02564.x

25. Raj N, Macmillan RD, Ellis IO, Deighton CM. Rheumatologists and breasts: Immunosuppressive therapy for granulomatous mastitis. Rheumatology (Oxford). 2004;43(8):1055-1056. doi:10.1093/rheuma tology/keh246

26. Oltean HN, Soliman AS, Omar OS, et al. Risk factors for chronic mastitis in Morocco and Egypt. Int J Inflam. 2013;2013:184921. doi:10.1155/ 2013/184921

27. Al-Khaffaf B, Knox F, Bundred NJ. Idiopathic granulomatous mastitis: A 25-year experience. J Am Coll Surg. 2008;206(2):269-273. doi:10. 1016/j.jamcollsurg.2007.07.041

28. Dobinson HC, Anderson TP, Chambers ST, Doogue MP, Seaward L, Werno AM. Antimicrobial treatment options for granulomatous mastitis caused by corynebacterium species. J Clin Microbiol. 2015;53(9): 2895-2899. doi:10.1128/JCM.00760-15

29. Ringsted S, Friedman M. A rheumatologic approach to granulomatous mastitis: A case series and review of the literature. Int J Rheum Dis. 2021;24(4):526-532. doi:10.1111/1756-185X.14065 